\section{Prevenção do Diabetes Mellitus Tipo 2}

\section{RESUMO}

Diabetes mellitus do tipo 2 (DM2) é uma doença metabólica complexa, multifatorial e de presença global, que afeta a qualidade e o estilo de vida dos acometidos, podendo levar a uma redução pronunciada na expectativa de vida dessa população. Portadores de diabetes podem ter uma redução de 15 ou mais anos de vida, com a grande maioria morrendo em decorrência das complicações cardiovasculares. Faz-se necessário o estabelecimento de estratégias efetivas para a redução do impacto do DM2 para os próximos anos. Para isso, urge a necessidade de maior atenção no tocante às estratégias de prevenção, sobretudo para as populações de maior risco de desenvolvimento da doença. Nesse contexto, os portadores de tolerância diminuída à glicose (TDG) e glicemia de jejum alterada (GJA) devem, cada vez mais, ser alvos de estratégias de intervenção na busca de minimização de risco para o diabetes, devendo para isso terem direcionamento para a efetivação de mudanças comportamentais (fatores dietoterápicos e prática de atividade física) e, quando necessário e aprovado, o uso de agentes farmacológicos. Estudos conduzidos pelo mundo têm confirmado a eficácia do uso de estratégias comportamentais e mesmo do uso de agentes farmacológicos para a prevenção de DM2. (Arq Bras Endocrinol Metab 2006;50/2:239-249)

Descritores: Prevenção; Diabetes tipo 2; Estilo de vida; Intervenção; Terapia farmacológica

\section{ABSTRACT}

Prevention of Type 2 Diabetes Mellitus.

Type 2 Diabetes mellitus (DM2) is a complex, multifactorial and worldwide metabolic disease that affects quality and lifestyle of patients. Patients with diabetes can have a 15-year or more reduction of lifetime and the high mortality is due to cardiovascular (CV) complications. Effective strategies for the reduction of the impact of DM2 on CV disorders for the next years and attention concerning to strategies of prevention, mainly for the populations with larger risk of developing the disease, are essential. In that context, impaired glucose tolerance (IGT) and impaired fasting glycemia (IFG) must suffer intervention strategies in order to minimize the risk for diabetes. Behavior modification (diet therapy and physical activity) must be stimulated, and pharmacological agents have to be used when indicated. Studies worldwide have been confirming the effectiveness of lifestyle strategies and even the use of pharmacological agents for the prevention of DM2. (Arq Bras Endocrinol Metab 2006;50/2:239-249)

Keywords: Prevention; Type 2 diabetes; Lifestyle; Intervention; Pharmacological therapy atualização

\author{
Ruy Lyra \\ Mônica Oliveiva \\ Daniel Lins \\ Ney Cavalcanti
}

Disciplina de Endocrinologia e Metabologia da Faculdade de Ciências Médicas de Pernambuco,

Universidade de Pernambuco, Recife, PE.
Recebido em 20/12/05

Aceito em 17/01/06 
D IABETES É DOENÇA CRÔNICA extremamente presente, afetando atualmente aproximadamente 171 milhões de indivíduos em todo o mundo e com projeção de alcançar 366 milhões de pessoas no ano de 2030, pulando a prevalência de 2,8\% em 2000 para 4,4\% (1). Números da Organização Mundial da Saúde (OMS) estimam que, em todo o globo, 987.000 mortes no ano de 2002 ocorreram por conta do diabetes, representando $1,7 \%$ da mortalidade geral (2). Dados recentemente publicados, utilizando um outro modelo de relação entre incidência, prevalência e mortalidade específica da doença, indicaram que o excesso de mortalidade global atribuível ao diabetes no ano de 2000 foi estimado em 2,9 milhões de mortes, equivalente a $5,2 \%$ da mortalidade geral, sendo $2-3 \%$ nos países pobres e mais de $8 \%$ em países desenvolvidos, tais como os Estados Unidos e Canadá (3). Esse quadro se tornará cada vez mais grave, em função da projeção de aumento pronunciado no número de acometidos. No tocante à perspectiva de grande incremento no número de portadores de diabetes tipo 2 (DM2), a susceptibilidade genética não pode justificar isoladamente esse quadro, sendo indubitavelmente os fatores ambientais parte fundamental desse cenário.

O diabetes traz também um grande impacto econômico para as nações. Só nos Estados Unidos, por exemplo, os custos diretos e indiretos com a doença em 2002 foram estimados em 132 bilhões de dólares (4). Apesar da introdução de novas drogas e da melhor compreensão dessa entidade clínica nos últimos anos, o controle dessa doença permanece insatisfatório na grande maioria da população. Dados americanos, analisando os níveis de $\mathrm{HbAlc}$, evidenciaram que menos de $50 \%$ dos portadores de diabetes mantêm sua doença adequadamente tratada, tendo havido até piora no controle metabólico, quando comparados os anos de 1988-94 (44,5\%) e 1999$2000(35,8 \%)(5)$.

Reduzir o impacto do DM2 significa, antes de tudo, reduzir a incidência da doença, antecipando-se ao seu aparecimento com medidas preventivas, sobretudo em indivíduos de alto risco, tais como os portadores de tolerância diminuída à glicose (TDG) e de glicemia de jejum alterada (GJA). Intervenções comportamentais e farmacológicas têm sido estudadas e implementadas com esse objetivo. Modificações no estilo de vida, tais como controle dietoterápico e prática sistemática de exercícios físicos, bem como o uso de alguns agentes orais, têm se mostrado eficazes. Aqui serão discutidos os principais estudos direcionados para a prevenção do DM2.

\section{FATORES DE RISCO MODIFICÁVEIS PARA O DM2}

Embora idade, histórico familiar, dentre outros fatores não modificáveis, possam estar presentes, na realidade os fatores modificáveis para o DM2 é que devem ser alvo de intervenção. Dentre os fatores de risco modificáveis para o DM2 destacam-se a obesidade e fatores dietoterápicos, o sedentarismo $(6,7)$, bem como o tabagismo. Stress psicossocial e episódios depressivos maiores também podem estar associados a um aumento de risco para DM2 (8).

\section{Obesidade}

A presença de sobrepeso/obesidade é situação cada vez mais presente no mundo atual $(9,10)$. O advento da obesidade é seguido de incremento significativo no risco para o desenvolvimento de DM2 $(6,11,12)$, e a resistência insulínica é um importante elo de ligação entre o DM2 e a obesidade. Portadores de obesidade abdominal, com maior deposição de gordura visceral característica, apresentam maior risco para desenvolvimento de DM2 (13). A gordura visceral apresenta um alto turnover metabólico, com expressiva atividade lipolítica, drenando expressivas concentrações de ácidos graxos livres (AGL) diretamente no fígado através da veia portal. Os AGL a nível hepático, por sua vez, reduzem o clearance da insulina e aumentam a produção hepática de glicose. Toda a ação que vise a perda ponderal, portanto, tende a reduzir a progressão dos quadros de TDG e GJA para DM2.

\section{Fatores dietoterápicos}

A composição alimentar parece ter influência na incidência de DM2. Um estudo analisando não diabéticos, realizado no Reino Unido, observou que a freqüência no consumo de frutas e vegetais esteve inversamente relacionada aos níveis de HbAlc (14). Um outro estudo, comparando indivíduos utilizando alto consumo de frutas, vegetais, peixes, aves e grãos, denominada dieta prudente, em relação àqueles utilizando a clássica dieta ocidental (alimentos industrializados, carne vermelha, alimentos ricos em gordura etc.), encontrou uma redução significativa de risco para desenvolvimento de DM2 naqueles fazendo uso da dieta prudente (15). Indivíduos ingerindo a dieta de padrão ocidental tiveram maior propensão para o desenvolvimento de DM2, independentemente do índice de massa corpórea (IMC), grau de atividade física, idade ou história familiar. Um outro campo de observação é o do papel de determinados macronutrientes na relação com o DM2. Estudo avaliando 41.254 americanos do sexo masculino, de diferentes grupos étnicos, en- 
controu uma relação entre o aumento de $40 \mathrm{~g}$ no consumo de gordura e incremento de risco no desenvolvimento de TDG e DM2, não se estabelecendo, entretanto, se esse fato foi mediado pelo elevada ingesta de gordura per si ou pela presença de obesidade (16).

\section{Sedentarismo}

A prática de atividade física de forma sistemática traz benefícios na musculatura esquelética, com mais eficiente uso da energia. A realização continuada de exercícios físicos aumenta a concentração de enzimas mitocondriais nos miócitos, o número de fibras musculares de contração lenta, assim como gera o desenvolvimento de capilares musculares (17). Além disso, o exercício físico age no processo de translocação dos transportadores de glicose (GLUT-4), essa ação parecendo justificar, ao menos em parte, os benefícios observados na sensibilidade insulínica no miócito (18). Portanto, via diferentes mecanismos, o exercício aumenta a captação de glicose mediada pela insulina na musculatura esquelética, e com isso melhora a sensibilidade insulínica $(19,20)$.

Vários estudos evidenciam que o aumento da atividade física reduz o risco de desenvolvimento de diabetes (21-23). Estudo prospectivo acompanhou 5.990 indivíduos do sexo masculino por 10 anos e avaliou o desenvolvimento de diabetes. Foi observado que o risco relativo para o aparecimento de diabetes foi significativamente menor naqueles que realizavam atividade física regular, mesmo quando ajustado para hipertensão e história familiar (24). Atividade física de moderada intensidade também reduziu a incidência de novos casos de DM2 em 900 finlandeses de meiaidade durante seguimento de aproximadamente 4,2 anos: observou-se um risco relativo de 0,44 nos praticantes quando comparados aos sedentários (25).

\section{Tabagismo}

Não há uma relação causal direta entre cigarro e diabetes, apesar de vários estudos prospectivos $(26,27)$ terem mostrado aumento da incidência de DM2 entre os fu- mantes. Estudos demonstraram que o cigarro aumenta a concentração da gordura a nível abdominal (28), reduz a sensibilidade insulínica (29) e eleva demasiadamente a concentração glicêmica após um teste oral de tolerância à glicose (30). O risco parece ser relacionado com a quantidade de cigarros e a duração do tabagismo. Estimativas do Physician's Health Study sugerem que, nos Estados Unidos, onde aproximadamente 25\% das pessoas são tabagistas, cerca de $10 \%$ da incidência do diabetes pode ser atribuída ao tabagismo (31).

Estudo prospectivo, que envolveu 7.735 homens com idade entre 40 e 59 anos, avaliou o impacto da interrupção do cigarro quanto ao risco de desenvolvimento de DM2. Os benefícios se tornaram evidentes 5 anos após a interrupção do tabagismo, no entanto o risco só foi revertido para o cenário dos nunca fumantes após 20 anos (32).

\section{Stress psicossocial}

O stress é um possível fator deletério para o desenvolvimento de DM2 (33). Em estudo americano, indivíduos com baixo nível de suporte emocional tiveram um incremento de risco para diabetes quando da presença de evento estressante não desejado, quando comparados com aqueles com altos níveis de suporte emocional (34).

\section{ESTRATÉGIAS DE PREVENÇĀO}

Uma vez bem conhecidos vários dos fatores de risco modificáveis para o desenvolvimento do DM2, uma série de programas de prevenção vem sendo testada. Esses programas, aplicados sob a forma de estudos, têm como base a modificação comportamental, o uso de fármacos e o tratamento cirúrgico.

\section{Intervenção no estilo de vida}

Vários estudos demonstraram que a mudança comportamental é eficaz como prevenção para o desenvolvimento de DM2 (tabela 1 ). Um dos primeiros

Tabela 1. Sumário dos principais estudos envolvendo mudança no estilo de vida.

\begin{tabular}{|c|c|c|c|c|}
\hline Estudo & $\begin{array}{l}\text { Número de } \\
\text { pessoas }\end{array}$ & $\begin{array}{l}\text { Duração } \\
\text { (anos) }\end{array}$ & Tipo de intervenção & $\begin{array}{c}\text { Redução da incidência } \\
\text { de diabetes } \\
\text { (pró intervenção) }\end{array}$ \\
\hline Malmo (35) & 181 & 6 & Dieta, exercício & $37 \%$ \\
\hline Da King (37) & 577 & 6 & $\begin{array}{c}\text { Dieta, exercício } \\
\text { ou ambos }\end{array}$ & $\begin{array}{c}31 \% \text { (dieta) } \\
46 \% \text { (exercícios) } \\
42 \% \text { (ambos) }\end{array}$ \\
\hline DPS (38) & 522 & 3,2 & Dieta, exercício & $58 \%$ \\
\hline DPP (39) & 3234 & 2,8 & Dieta, exercício & $58 \%$ \\
\hline
\end{tabular}


estudos que visava avaliar o impacto da mudança comportamental para a prevenção do DM2 foi o 6year Malmo Feasibility Study (35). Nesse estudo, de aproximadamente 6 anos de seguimento, foram selecionados portadores de DM2 recentemente diagnosticados e indivíduos com TDG, sendo esses comparados com indivíduos normoglicêmicos. Parte dos portadores de TDG e os indivíduos com DM2 foram submetidos a tratamento dietoterápico e atividade física sistemática. Ao final do estudo, o peso corporal foi reduzido em $2,3-7 \%$ nos participantes submetidos à mudança comportamental, enquanto os indivíduos com TDG sem intervenção e os controles normais ganharam peso $(0,5-1,7 \%)$. Como resultado, a normalização do teste oral de tolerância à glicose (TOTG) foi encontrada em mais de $50 \%$ dos portadores de TDG sob intervenção, bem como mais de $50 \%$ dos portadores de DM2 entraram em remissão após 6 anos de seguimento. A melhora na tolerância à glicose foi correlacionada à redução ponderal $(\mathrm{r}=0,19, \mathrm{p}<$ $0,02)$ e atividade física $(\mathrm{r}=0,22, \mathrm{p}<0,02)$. Desse mesmo grupo de indivíduos surgiu o estudo Malmo Preventive Trial (36), que foi desenvolvido para comparar as taxas de mortalidade cardiovascular e geral em portadores de TDG sob intervenção comportamental, em comparação com indivíduos normoglicêmicos. Portadores de TDG foram submetidos a um programa de mudança comportamental (programa dietoterápico e de exercícios físicos regulares) durante 12 anos de acompanhamento. Como resultado, os portadores de TDG sob intervenção tiveram risco similar aos normoglicêmicos, tanto para mortalidade cardiovascular como para mortalidade por todas as causas $(6,5$ vs. 6,2 por 1.000 pessoas/ano de risco). O risco para esses desfechos foi muito inferior ao observado em pacientes com TDG sem intervenção $(6,5$ vs. 14 por 1.000 pessoas/ano de risco, $\mathrm{p}=$ 0,009 ), sendo essa redução correlacionada com a redução ponderal e com a atividade física.

O Da Qing Study (37) visava observar o papel de prevenção para o desenvolvimento de DM2 em portadores de TDG frente a mudanças comportamentais de forma isolada (modificação dietoterápica ou atividade física) ou de forma agrupada (dieta e atividade física), em comparação com indivíduos controles. Após 6 anos de seguimento, a incidência cumulativa de DM2 no grupo controle foi de $67 \%$. No tocante aos grupos de intervenção em comparação ao grupo controle, a redução de desenvolvimento de DM2 foi de $31 \%$ no grupo de dieta isolada ( $\mathrm{p}<0,003), 46 \%$ no grupo de atividade física isolada $(\mathrm{p}<0,005)$ e $42 \%$ no grupo de dieta + exercícios $(\mathrm{p}<0,005)$.
O Finnish Diabetes Prevention Study (DPS) (38) visava avaliar 522 portadores de TDG, com idade média de 55 anos, randomizados em 2 grupos: grupo controle e grupo intervenção. Cada indivíduo do grupo intervenção recebia aconselhamento individualizado acerca da necessidade de redução ponderal, redução na ingesta de gordura, sobretudo a gordura saturada, incremento na ingesta de fibras e prática de atividade física regular. $\mathrm{O}$ tempo médio de seguimento foi de 3,2 anos, onde a incidência cumulativa de diabetes foi de $11 \%$ (95\% intervalo de confiança, 6-15\%) no grupo intervenção e $23 \%$ (95\% intervalo de confiança, 17-29\%) no grupo controle, reduzindo em $58 \%$ o risco de desenvolvimento de DM2 nos indivíduos do grupo de intervenção $(p<0,001)$. Ao fim de 2 anos de seguimento, o grupo intervenção tinha perdido uma média de $3,5 \mathrm{~kg}$. Foi observado que a redução na incidência de diabetes foi diretamente associada com as mudanças no estilo de vida.

O Diabetes Prevention Program (DPP) (39) foi um estudo que avaliou 3.234 obesos (IMC médio de $34 \mathrm{~kg} / \mathrm{m}^{2}$ ) com TDG, subdividindo-os em 3 grupos: o primeiro de modificações no estilo de vida (aconselhamento para dieta e exercícios, com prática de pelo menos 150 minutos de atividade física por semana e perda ponderal de, ao menos, $7 \%$ do peso inicial), o segundo utilizando a metformina $(850 \mathrm{mg}$ duas vezes ao dia) e o terceiro sendo o grupo controle. $\mathrm{O}$ acompanhamento médio foi de 2,8 anos. A incidência de diabetes foi de 11 casos por 100 pessoas/ ano no grupo placebo, 7,8 casos por 100 pessoas/ano no grupo metformina e 4,8 casos por 100 pessoas/ano no grupo de intervenção comportamental. O grupo de intervenção comportamental, portanto, reduziu em $58 \%$ a incidência de DM2 (RR 0,42, 95\% intervalo de confiança, $48-66 \%$ ), enquanto a metformina reduziu $31 \%$ (RR 0,69, 95\% intervalo de confiança, 17-43\%), ambos quando comparados ao grupo controle. No grupo de modificações no estilo de vida, mais de $50 \%$ dos pacientes alcançaram a meta de perda ponderal superior a $7 \%$, e $74 \%$ mantiveram ao menos 150 minutos de atividade física semanal. Para prevenir um caso de DM2 durante o período de 3 anos, 6,9 indivíduos necessitariam participar de um programa de mudança comportamental, enquanto 13,9 necessitariam utilizar a metformina.

Meta-análise recente, avaliando estudos cujas intervenções foram modificações no estilo de vida (dieta e exercício), evidenciou que a redução da glicose plasmática após o TOTG foi de aproximadamente $0,84 \mathrm{mmol} / \mathrm{l}(15 \mathrm{mg} / \mathrm{dl})$ e a incidência de diabetes foi reduzida em aproximadamente $50 \%$ quando comparado com placebo (40). 


\section{Intervenção farmacológica}

Uma vez que nem sempre é possível se estabelecer uma mudança comportamental plena na população de alto risco, e mesmo tentando ampliar o papel de proteção contra o desenvolvimento de DM2, uma série de fármacos vem sendo testada com o intuito de prevenção dessa doença. Várias drogas já foram utilizadas, com alguns dos principais estudos sendo aqui sumarizados. Destacam-se fármacos tais como anti-diabéticos orais e outros agentes orais.

\section{Anti-diabéticos orais}

Vários são os anti-diabéticos orais testados com o objetivo de prevenção do DM2 (tabela 2). Os principais estudos serão apresentados de acordo com a classe do fármaco.

\section{Sulfoniluréias}

Dois estudos examinaram o efeito da tolbutamida na incidência de diabetes em portadores de TDG ou naqueles com glicemia plasmática nos níveis superiores de normalidade $(41,42)$. Nenhum dos estudos evidenciou redução significativa na incidência de DM2 quando comparado com o grupo placebo.

\section{Acarbose}

A acarbose, um inibidor da alfa-glicosidase, vem sendo testada na prevenção da DM2. No estudo STOPNIDDM, 1.429 pacientes com TDG foram randomizados para receber acarbose $100 \mathrm{mg}, 3$ vezes ao dia, ou placebo, por um período de 3,3 anos. A acarbose demonstrou uma redução significativa de $25 \%$ no risco relativo de progressão para diabetes quando comparada ao placebo. Se o diagnóstico de TDG foi confirmado com um segundo TOTG, a redução do risco relativo para o desenvolvimento de DM2 no grupo da acarbose foi de $36 \%$. A acarbose foi efetiva em todos os grupos de pacientes, entretanto mulheres com mais de 55 anos, portadoras de índice de massa corpórea (IMC) $<30 \mathrm{~kg} / \mathrm{m}^{2}$, bem como com pressão arterial e concentrações de insulina plasmática normais, tiveram um maior benefício (43-45). Quando esta medicação foi interrompida, houve um aumento na incidência de diabetes, sugerindo que o benefício desta droga só ocorre durante a sua utilização. Como vantagens adicionais, os pacientes em utilização da acarbose tiveram significativos efeitos de proteção vascular, tais como reduções de $49 \%$ no risco relativo de desenvolvimento de eventos cardiovasculares e $34 \%$ na incidência de novos casos de hipertensão (46).

\section{Biguanidas}

A fenformina foi a primeira biguanida testada para a prevenção do DM2. No Whitehall Study, a fenformina não produziu benefícios de proteção quanto ao desenvolvimento de DM2 em portadores de TDG (47). O mesmo fato foi observado em outro estudo com o mesmo fármaco (48). No entanto, o maior e mais rigoroso estudo do ponto de vista metodológico com essa classe de drogas foi conduzido com o uso da metformina. O Diabetes Prevention Programme (DPP) randomizou 2.155 indivíduos portadores de TDG para intervenção comportamental, metformina ou placebo. Após um seguimento médio de 2,8 anos, a incidência de diabetes foi de $7,8 \%$ no grupo tratado com placebo versus $4,8 \%$ nos tratados com metformina (RR $0,69,95 \%$, intervalo de confiança $0,57-0,83)$. O uso da metformina também se acompanhou de uma redução ponderal de $2 \mathrm{~kg}$, sendo mais efetiva em pacientes com menos de 44 anos e com IMC > $35 \mathrm{~kg} / \mathrm{m}^{2}$ (39). Alguns outros estudos com o uso das biguanidas não mostraram reduções significativas na incidência de diabetes quando comparados com placebo; entretanto, todos mostravam poucos casos de diabetes para os diferentes grupos analisados $(49,50)$.

\section{Thiazolidinedionas}

A eficácia das glitazonas na prevenção da DM2 foi primeiro demonstrada no TRIPOD Study, que acompanhou um grupo de 235 mulheres hispânicas com histórico de diabetes gestacional prévia. Essas foram randomizadas para receber troglitazona $(400 \mathrm{mg} / \mathrm{dia})$

Tabela 2. Sumário de alguns dos principais estudos envolvendo anti-diabéticos orais.

\begin{tabular}{lcccc}
\hline Estudo & $\begin{array}{c}\text { Número de } \\
\text { pessoas }\end{array}$ & $\begin{array}{c}\text { Duração } \\
\text { (anos) }\end{array}$ & $\begin{array}{c}\text { Tipo de intervenção } \\
\text { (em comparação } \\
\text { com placebo) }\end{array}$ & $\begin{array}{c}\text { Redução da incidência } \\
\text { de diabetes } \\
\text { (pró-intervenção) }\end{array}$ \\
\hline STOP-NIDDM (43) & 1.429 & 3,3 & Acarbose & $25 \%$ \\
Jarrett e cols. (47) & 204 & 5,0 & Fenformina & $10 \%$ \\
DPP (39) & 2.155 & 2,8 & Metformina & $31 \%$ \\
TRIPOD (51) & 235 & 2,5 & Troglitazona & $56 \%$ \\
DPP (52) & 585 & 0,9 & Troglitazona & $75 \%$ \\
Durbin e cols. (53) & 101 & 3,0 & Rosiglitazona/Pioglitazona & $88,9 \%$ \\
\hline
\end{tabular}


ou placebo. Após 2,5 anos de seguimento, a incidência anual de DM2 foi $12,3 \%$ no grupo placebo e $5,4 \%$ na troglitazona, o equivalente a uma redução de risco para progressão para DM2 de $56 \%$. Oito meses após a interrupção do estudo e da troglitazona, a proteção na prevenção de DM2 ainda era vista (51). A troglitazona também foi um das drogas inicialmente selecionadas para uso no estudo DPP, tendo, no entanto, sido suspensa após 11 meses de seguimento, em decorrência de casos de toxidade hepática em alguns pacientes randomizados para seu uso. Estudo recentemente publicado analisou o impacto do uso da troglitazona no estudo DPP ao longo dos 9 meses de sua utilização (52). Quando comparado ao grupo placebo, a troglitazona reduziu em 75\% a incidência de DM2, enquanto a metformina e a modificação intensiva no estilo de vida obtiveram reduções de $44 \%$ e $58 \%$ respectivamente. Um outro estudo visou analisar a capacidade de prevenção para DM2 com a rosiglitazona e a pioglitazona (53). Cento e um pacientes que tinham recebido troglitazona por aproximadamente 10 meses, após a sua retirada do mercado americano, foram transferidos para tratamento ativo com rosiglitazona (4 mg/dia) ou pioglitazona ( $30 \mathrm{mg} / \mathrm{dia})$. Pacientes com TDG e resistência à insulina sem uso de antidiabéticos orais serviram de grupo controle. Como resultado, após 2 anos de tratamento, nenhum dos pacientes recebendo rosiglitazona ou pioglitazona progrediu para DM2, enquanto 11 pacientes do grupo controle tornaram-se diabéticos. Com 3 anos de seguimento, 3 pacientes dos grupos das glitazonas desenvolveram DM2, em comparação com 19 pacientes do grupo controle. Portanto, a redução de risco para aparecimento de DM2 no grupo em uso das glitazonas após 3 anos de seguimento foi de $88,9 \%$ quando comparado com o grupo controle $(\mathrm{p}<0,001)$. Estudos maiores com a rosiglitazona e a pioglitazona estão sendo conduzidos para tentar demonstrar o papel desses fármacos na prevenção do DM2 (54)

\section{Outros agentes orais}

Vários outros agentes orais têm sido utilizados para testar eficácia na prevenção de DM2 (tabela 3). Destacam-se agentes anti-obesidade, anti-hipertensivos, estatinas, fibratos e estrógenos.

\section{Agentes anti-obesidade}

Já é bem estabelecido que o risco de desenvolvimento de DM2 é intimamente correlacionado à presença e duração de sobrepeso e obesidade. Drogas antiobesidade foram, portanto, utilizadas para se testar a possibilidade de se prevenir o DM2. No Xenical in the Prevention of Diabetes in Obese Subjects study (XENDOS), o orlistat $120 \mathrm{mg}$ três vezes ao dia versus placebo foi utilizado em 3.305 indivíduos portadores de obesidade (IMC $>30 \mathrm{~kg} / \mathrm{m}^{2}$ ), apresentando normalidade glicêmica (59\%) ou TDG $(21 \%)$ por aproximadamente 4 anos. Ao final do período de observação, a incidência cumulativa de diabetes foi de $9,0 \%$ com placebo e $6,2 \%$ com orlistat, correspondendo a uma redução de risco de $37,7 \%(\mathrm{p}=0,0032)$. A média de perda ponderal foi maior no grupo em uso do orlistat ( 5,8 vs. $3,0 \mathrm{~kg}$ com placebo, $\mathrm{p}<0,001)(55)$. Entretanto, uma análise conjunta de três diferentes estudos clínicos envolvendo 642 pacientes obesos demonstrou uma redução não significativa na incidência de DM2 de 2,0 para $0,6 \%$ no grupo fazendo uso de orlistat (RR $0,25,95 \%$ intervalo de confiança $0,05-1,2)$ quando comparado com placebo (56).

\section{Drogas anti-hipertensivas}

Drogas que não são reconhecidas como anti-diabétcos orais têm mostrado resultados interessantes no tocante à prevenção do DM2. Nesse grupo incluem-se alguns agentes anti-hipertensivos. Uma revisão feita em 24 estudos envolvendo drogas anti-hipertensivas encontrou que a incidência de diabetes não é modificada ou mesmo aumentada quando do uso de diuréticos tiazídicos e $\beta$ bloqueadores, podendo, entretanto, não ser

Tabela 3. Sumário de alguns dos principais estudos envolvendo outros agentes orais.

\begin{tabular}{lcccc}
\hline Estudo & $\begin{array}{c}\text { Número de } \\
\text { pessoas }\end{array}$ & $\begin{array}{c}\text { Duração } \\
\text { (anos) }\end{array}$ & $\begin{array}{c}\text { Tipo de intervenção } \\
\text { (em comparação } \\
\text { com placebo) }\end{array}$ & $\begin{array}{c}\text { Redução da incidência } \\
\text { de diabetes } \\
\text { (pró-intervenção) }\end{array}$ \\
\hline XENDOS (55) & 3.305 & 4,0 & Orlistat & $37,7 \%$ \\
HOPE (60) & 5.720 & 4,5 & Ramipril & $34 \%$ \\
CHARM (62) & 3.803 & 2,0 & Candesartan & $22 \%$ \\
WOSCOPS (67) & 6.447 & 4,9 & Pravastatina & $30 \%$ \\
LIPID (68) & 6.997 & 6,0 & Pravastatina & $11 \%$ \\
HPS (69) & 14.573 & 5,0 & Sinvastatina & $+15 \%$ \\
BIP (71) & 303 & 6,2 & Bezafibrato & $30 \%$ \\
HERS (72) & 2.029 & 4,1 & Estrógeno + Progesterona & $35 \%$ \\
\hline
\end{tabular}


alterada ou mesmo reduzida com o uso de inibidores da enzima de conversão da angiotensina (IECA), bloqueadores do receptor da angiotensina (BRA) e bloqueadores do canal de cálcio (BCC) (57).

Os diuréticos tiazídicos foram associados com aumento não significativo da incidência de DM2 no Systolic Hypertension in the Elderly Program trial (SHEP) (RR 1,2, 95\% intervalo de confiança 0,9-1,5) (58) e no European Working Party on High Blood Pressure in the Elderly trial (EWHPE) (RR 1,5, 95\% intervalo de confiança $0,85-1,6)(59)$.

No Heart Outcomes Prevention Evaluation trial (HOPE), estudo que incluiu 5.720 pacientes acompanhados por aproximadamente 4,5 anos, aqueles utilizando o ramipril apresentaram uma redução significativa na incidência de DM2 de 5,4 para 3,6\% (RR 0,66, 95\% intervalo de confiança $0,51-0,85, \mathrm{p}<$ 0,001) (60). No Captopril Prevention Project, estudo que acompanhou 10.985 pacientes hipertensos, no grupo de pacientes recebendo o captopril, 6,5\% desenvolveram diabetes, comparado a $7,2 \%$ no grupo recebendo placebo $(\mathrm{p}<0,005)(61)$.

$\mathrm{O}$ uso dos BRA reduziu significativamente o aparecimento de DM2 no Candesartan in Heart Failure Assessment of Reduction in Mortality and Morbidity study (CHARM) de 7\% para 6\% (RR 0,78, 95\% intervalo de confiança 0,64-0,96) (62) e obteve redução não significativa no Study of Cognition and Prognosis in the elderly trial (SCOPE) (RR 0,81, intervalo de confiança 0,62-1,06) (63). O Losartan Intervention For Endpoint study (LIFE), acompanhando mais de 9.000 hipertensos em uso do losartan ou atenolol, demonstrou que o tratamento com losartan foi associado com menor risco de desenvolvimento de DM2 quando comparado com aqueles utilizando atenolol ( 13,0 por 1.000 vs. 17,5 por 1.000 pessoas / ano de tratamento) (64). O Verapamil-Trandolapril study (INVEST) analisou 16.176 pacientes hipertensos com doença coronariana prévia e encontrou uma redução na incidência de DM2 naqueles em uso do verapamil, quando comparados com os em uso do atenolol $(8,2 \%$ para $7,0 \%)$ ( RR 0,85 , intervalo de confiança $0,77-0,95)(65)$. O que não se sabe nesses dois últimos estudos é se a diferença é devida ao uso de losartan e verapamil, respectivamente, ou se ocorre pelo efeito deletério do atenolol no metabolismo dos carboidratos. Já o Valsartan Antibypertensive Longterm Use Evaluation trial (VALUE), onde foram avaliados 10.419 portadores de hipertensão com alto risco cardiovascular, os indivíduos alocados no grupo valsartan apresentaram uma redução de $16 \%$ para $13 \%$ na incidência de DM2 quando comparados àqueles utilizando amlodipina ( $\mathrm{RR}$ 0,77, 95\% intervalo de confiança 0,69-0,86) (66). Embora esses resultados sugiram um papel de proteção contra o desenvolvimento de DM2, nenhum dos estudos teve como objetivo primário a avaliação de redução de risco para aparecimento de diabetes, e os dados no momento são insuficientes para a recomendação desses grupos de drogas para pacientes de alto risco.

\section{Estatinas}

No tocante ao uso das estatinas e seus efeitos na incidência de diabetes, os resultados são conflitantes. No West of Scotland Coronary Prevention Study (WOSCOPS), a incidência de diabetes foi significativamente reduzida com o uso da pravastatina (RR 0,70, 95\% intervalo de confiança 0,50-0,99) (67) e no Longterm Intervention with Pravastatin in Ischemic Disease study (LIPID), 4,0\% dos pacientes tratados com pravastatina desenvolveram diabetes versus $4,5 \%$ no grupo placebo $(0,89,0,70-1,13)(68)$. Por outro lado, no Heart Protection Study (HPS), 4,6\% dos pacientes tratados com a sinvastatina desenvolveram DM2, comparado com 4,0\% daqueles em uso do placebo (69). Resultados similares foram vistos no Anglo-Scandinavian Cardiac Outcomes Trial - Lipid Lowering Arm (ASCOT-LLA), onde a incidência de diabetes naqueles fazendo uso da atorvastatina foi de 3,0\%, enquanto no grupo em uso do placebo foi de 2,6\% $(1,15,0,91-1,44)(70)$. Portanto, o papel das estatinas na prevenção de DM2 necessita de futuras avaliações prospectivas.

\section{Fibratos}

Poucos são os dados quanto aos possíveis efeitos de proteção dos fibratos quanto ao desenvolvimento de DM2. Entretanto, analisando 303 pacientes portadores de TDG incluídos no Bezafibrate Infarction Prevention trial (BIP), a terapia com bezafibrato foi associada com a redução da incidência de diabetes de $54 \%$ para $42 \%$ comparado com placebo (RR 0,70, 95\% intervalo de confiança $0,49-0,99)(71)$.

\section{Estrógenos}

Um estudo clínico randomizado e alguns estudos de coorte têm examinado a relação entre o uso de estrógenos e a ocorrência de diabetes. Análise post hoc do Estrogen/Progestin Replacement Study (HERS) indicou que a associação de estrógeno e progesterona foi associada com uma redução significativa na incidência de diabetes quando comparada com placebo (RR $0,65,95 \%$ intervalo de confiança $0,48-0,89)(72)$. O estudo Nurses Health Study, um grande estudo de 
coorte, demonstrou que as usuárias ativas de estrógenos foram associadas com uma significativa redução na incidência de diabetes quando comparadas àquelas que nunca fizeram uso (RR 0,82, 95\% intervalo de confiança $0,70-0,96)(73)$. Um outro estudo, examinando num seguimento de 12 anos a incidência de DM2 em 21.028 mulheres americanas na pós-menopausa e livres do diagnóstico de diabetes, doença cardiovascular e câncer, encontrou que aquelas em uso corrente de terapia de reposição hormonal tiveram uma redução no risco relativo para desenvolvimento de diabetes (RR 0,8,95\% intervalo de confiança $0,67-0,96)$ quando comparadas àquelas sem uso $(74)$. Embora alguns estudos sugiram que a terapia de reposição hormonal reduz a incidência de DM2, se recomenda esse tratamento como estratégia de prevenção de DM2 em mulheres pós-menopausa.

\section{Tratamento cirúrgico}

Uma das possíveis estratégias de prevenção para indivíduos obesos mórbidos é o uso da cirurgia bariátrica.

\section{Cirurgia bariátrica}

A cirurgia bariátrica é um dos tratamentos mais efetivos na prevenção da DM2, segundo alguns estudos. O Swedish Obese Subjects trial (SOS) comparou obesos mórbidos submetidos à cirurgia gástrica bariátrica versus controles (obesos mórbidos não operados, em tratamento convencional). Após 2 anos de seguimento, os obesos submetidos ao tratamento cirúrgico tinham perdido $28 \pm 15 \mathrm{~kg}$, em comparação a $0,5 \pm$ $8,9 \mathrm{~kg}$ no grupo controle, com os operados apresentando uma redução bastante significativa no desenvolvimento de DM2 (RR 0,02, 95\% intervalo de confiança $0,00-0,16)(75)$. Avaliação posterior, já com 8 anos de seguimento, continuou demonstrando um significativo efeito na redução da incidência de diabetes entre os submetidos à cirurgia bariátrica (OR $0,16,95 \%$ intervalo de confiança $0,07-0,36)$ quando comparados aos não operados (76). Um outro estudo conduzido em 163 obesos mórbidos com DM2 ou TDG e utilizando a técnica de bypass gástrico de Greenville, evidenciou que 141 (86\%) operados reverteram o quadro para normalidade glicêmica e somente 22 indivíduos permaneceram com alteração no metabolismo dos hidratos de carbono (77).

\section{CONCLUSÃO}

Uma série de estudos tem examinado o impacto da mudança comportamental e do uso de diferentes fármacos numa possível redução na incidência de diabetes. Tanto a perda ponderal quanto a atividade física sistemática se mostraram eficazes na prevenção do DM2. O tratamento cirúrgico, através da cirurgia bariátrica, se acompanhou de eficácia quanto à redução no desenvolvimento de DM2 em portadores de obesidade mórbida. Embora o uso de diversos fármacos, tais como antidiabéticos orais, agentes antiobesidade, anti-hipertensivos, estatinas, fibratos e estrógenos, tenha se acompanhado de redução na incidência de diabetes, futuros estudos são necessários para a recomendação dessas intervenções como prevenção para pacientes de alto risco.

\section{REFERÊNCIAS}

1. Wild S, Roglic G, Green A, Sicree S, King H. Global prevalence of diabetes - Estimates for the year 2000 and projections for 2030. Diabetes Care 2004;27:1047.

2. World Health Organization. The World Health Report 2003. Geneva:World Health Organization; 2003.

3. Roglic $G$, Unwin N, Bennett PH, Mathers C, Tuomilehto J, $\mathrm{Nag} S$, et al. The burden of mortality attributable to diabetes: realistic estimates for the year 2000. Diabetes Care 2005;28(9):2130-5.

4. American Diabetes Association. Economics costs of diabetes in US in 2002. Diabetes Care 2003:26:917-32.

5. Koro CE, Bowlin SJ, Bourgeois N, Fedder DO. Glycemic control from 1988 to 2000 among US adults diagnosed with type 2 diabetes: a preliminary report. Diabetes Care 2004;27(1):17-20.

6. Colditz GA, Willett WC, Rotnitzky A, Manson JE. Weight gain as a risk factor for clinical diabetes mellitus in women. Ann Intern Med 1995; 122:481-6.

7. Chan JM, Rimm EB, Colditz Stampfer MJ, Willet WC. Obesity, fat distribution and weight gain as risk factors for clinical diabetes in men. Diabetes Care 1994; 17:961-9.

8. Ramachandran A, Snehalatha C, Shobana R, Vidyavathi $P$, Vijay $V$. Influence of life-style factors in development of diabetes in Indians - scope for primary prevention. J Assoc Phys India 1999:47:761-3.

9. Kumanyika S, Jeffery RW, Morabia A, Ritenbaugh C, Antipatis VJ. Obesity prevention; the case for action. Int J Obes Relat Metab Disord 2002;26:425-36.

10. Golay A, Masciangelo ML. Burden of obesity: from epidemic to costs. Rev Med Suisse 2005; 1(12):807-10, 813.

11. Oster G, Edelsberg J, O'Sullivan AK, Thompson D. The clinical and economic burden of obesity in a managed care setting. Am J Manag Care 2000;6(6):681-9.

12. Mayer-Davis EJ, Costacou T. Obesity and sedentary lifestyle: modifiable risk factors for prevention of type 2 diabetes. Curr Diab Rep 2001;1(2):170-6. 
13. Chan JM, Rimm EB, Colditz GA, Stampfer MJ, Willett WC. Obesity, fat distribution, and weight gain as risk factors for clinical diabetes in men. Diabetes Care 1994; 17(9):961-9.

14. Sargeant LA, Khaw KT, Bingham S, Day NE, Luben RN, Oakes $S$, et al. Fruit and vegetable intake and population glycosylated haemoglobin levels: the EPIC-Norfolk Study. Eur J Clin Nutr 2001:55(5):342-8.

15. Van Dam R, Rimm E, Willett W, Stampfer M. Dietary patterns and risk for type 2 diabetes mellitus in US men. Ann Intern Med 2002; 136:201.

16. Choi HK, Willett WC, Stampfer MJ, Rimm E, Hu FB. Dairy consumption and risk of type 2 diabetes mellitus in men: a prospective study. Arch Intern Med 2005; 165(9): 997-1003.

17. Henriksson J. Effects of physical training on the metabolism of skeletal muscle. Diabetes Care 1992;15:1701-11.

18. Mcauley K, Williams S, Mann J, Goulding A, Chisholm A, Wilson $\mathrm{N}$, et al. Intensive lifestyle changes are necessary to improve insulin sensitivity: a randomized controlled trial. Diabetes Care 2002;25:445-52.

19. Annuzi G, Riccardi G, Capaldo S, Kaijser L. Increased insulin-stimulated glucose uptake by exercised human muscles one day after prolonged physical exercise. Euro J Clin Invest 1991;21:6-12.

20. Zawadzki JK, Bogardus C, Foley JE. Insulin action in obese noninsulin-dependent diabetics and in their isolated adipocytes before and after weight loss. Diabetes 1987;36:227-36

21. Burchfield CM, Sharp DS, Curb JD, Rodriguez, Hwang L$\mathrm{J}$, Marcus EB, et al. Physical activity and incidence of diabetes: The Honolulu Heart Program. Am J Epidemiol $1995 ; 141: 360-8$

22. Manson JE, Nathan DM, Krolewski AS, Stampfer MJ, Willett WC, Hennekens $\mathrm{CH}$. A prospective study of exercise and incidence of diabetes among US male physicians. JAMA 1992;268:63-7.

23. Perry IJ, Wannamethee SG, Walker MK, Thomson AG, Whincup PH, Shaper AG. Prospective study of risk factors for development of non-insulin dependent diabetes in middle-aged British men. Br Med J 1995;310:560-4.

24. Helmrich SP, Ragland DR, Leung RW, Paffenbarger RS Jr. Physical activity and reduced occurrence of non-insulindependent diabetes mellitus. N Engl J Med 1991;325(3): 147-52.

25. Lynch J, Helmrich SP, Lakka TA, Kaplan GA, Cohen RD, Salonen $\mathrm{R}$, et al. Moderately intense physical activities and high levels of cardiorespiratory fitness reduce the risk of non-insulin-dependent diabetes mellitus in middleaged men. Arch Intern Med 1996;156:1307-14.

26. Rimm EB, Chan J, Stampfer MJ, Colditz GA, Willett WC. Prospective study of cigarette smoking, alcohol use, and the risk of diabetes in men. BMJ 1995;310:555-9.

27. Uchimoto S, Tsumura K, Hayashi T, Suematsu C, Endo G, Fujii $S$, et al. Impact of cigarette smoking on the incidence of type 2 diabetes mellitus in middle-aged Japanese men: the Osaka Health Survey. Diabet Med 1999; 16:951-5.
28. Shimokata H, Muller DC, Andres R. Studies in the distribution of body fat. III. Effects of cigarette smoking. JAMA 1989:261:1169-73.

29. Frati AC, Iniestra F, Ariza CR. Acute effect of cigarette smoking on glucose tolerance and other cardiovascular risk factors. Diabetes Care 1996; 19:112-8.

30. Janzon L, Berntorp K, Hanson M, Lindell SE, Trell E. Glucose tolerance and smoking: a population study of oral and intravenous glucose tolerance tests in middleaged men. Diabetologia 1983:25:86-8.

31. Manson JE, Ajani UA, Liu S, Nathan DM, Hennekens $\mathrm{CH}$. A prospective study of cigarette smoking and the incidence of diabetes mellitus among US male physicians. Am J Med 2000; 109:538-42.

32. Wannamethee SG, Shaper AG, Perry IJ; British Regional Heart Study. Smoking as a modifiable risk factor for type 2 diabetes in middle-aged men. Diabetes Care 2001; 24:1590-5.

33. Ramachandran A, Snehalatha C, Shobana R, Vidyavathi P, Vijay V. Influence of life-style factors in development of diabetes in Indians - scope for primary prevention. J Assoc Physicians India 1999:47:761-3.

34. Krause N. Stress and diabetes mellitus in later life. Int J Aging Hum Dev 1995;40:125-43.

35. Eriksson KF, Lindgarde F. Prevention of type 2 diabetes mellitus by diet and physical exercise. The 6-year Malmo feasibility study. Diabetologia 1991;34:891-8.

36. Eriksson K-F, Lindgarde F. No excess 12-year mortality in men with impaired glucose tolerance who participated in the Malmo Preventive Trial with diet and exercise. Diabetologia 1998;41:1010-6.

37. Pan XR, Li GW, Hu YH, Wang JX, Yang WY, An ZX, et al. The Da Qing IGT, Diabetes Study. Effects of diet and exercise in preventing NIDDM in people with impaired glucose tolerance. Diabetes Care 1997;20:537-44.

38. Tuomilehto J, Lindstrom J, Eriksson JG, Valle TT, Hamalainen $\mathrm{H}$, llanne-Parikka $\mathrm{P}$, et al. Prevention of type 2 diabetes by changes in lifestyle among subjects with impaired glucose tolerance. N Engl J Med 2001: 344:1343-50.

39. Knowler WC, Barrett-Connor E, Fowler SE, Hamman RF, Lachin JM, Walker EA, et al; Diabetes Prevention Program Research Group. Reduction in the incidence of type 2 diabetes with lifestyle intervention or metformin. $\mathbf{N}$ Engl J Med 2002;346:393-403.

40. Kazua Y, Tongo T. Efficacy of lifestyle education to prevent type 2 diabetes: a meta-analysis of randomized controlled trials. Diabetes Care 2005:28:2780-6.

41. Sartor G, Schersten B, Carlstrom S, Melander A, Norden A, Persson G. Ten-year follow-up of subjects with impaired glucose tolerance: prevention of diabetes by tolbutamide and diet regulation. Diabetes 1980;29:41-9.

42. Keen $\mathrm{H}$, Jarrett RJ, Ward JD, Fuller JH. Borderline diabetics and their response to tolbutamide. Adv Metab Disord 1973:2(suppl 2):521-31.

43. Chiasson JL, Gomis R, Hanefeld M, Josse RG, Karasik A, Laakso M; The STOP-NIDDM Trial Research Group. The STOP-NIDDM Trial. An international study on the efficacy of an a-glucosidase inhibitor to prevent type 2 diabetes in a population with impaired glucose tolerance: rationale, design, and preliminary data. Diabetes Care $1998: 21: 1720-5$ 
44. Chiasson JL, Josse RG, Gomis R, Hanefeld M, Karasik A, Laakso M. Acarbose for prevention of Type 2 diabetes mellitus: the STOP-NIDDM randomized trial. Lancet 2002;359:2072-7.

45. Hanefeld M, Josse RG, Gomis R, Karasik A, Laakso M, Chiasson JL. Efficacy of acarbose to prevent type 2 diabetes is different in subgroups of subjects with impaired glucose tolerance: The STOP-NIDDM trial. Diabetologia 2002;45:S104-5.

46. Chiasson JL, Josse RG, Gomis R, Hanefeld M, Karasik A Laakso M; STOP-NIDDM Trial Research Group. Acarbose treatment and the risk of cardiovascular disease and hypertension in patients with impaired glucose tolerance: the STOP-NIDDM trial. JAMA 2003;290:486-94.

47. Jarrett RJ, Keen H, Fuller JH, McCartney M. Treatment of borderline diabetes: controlled trial using carbohydrate restriction andphenformin. Br Med J 1977;2:861-5.

48. Jarrett RJ, Keen H, Fuller JH, McCartney M. Worsening to diabetes in men with impaired glucose tolerance ("borderline diabetes"). Diabetologia 1979;16:25-30.

49. Li CL, Pan CY, Lu JM, Zhu Y, Wang JH, Deng XX, et al. Effect of metformin on patients with impaired glucose tolerance. Diabet Med 1999;16:477-81.

50. Fontbonne A, Charles MA, Juhan-Vague I, Bard JM, Andre $\mathrm{P}$, Isnard $\mathrm{F}$, et al. The effect of metformin on the metabolic abnormalities associated with upper-body fat distribution. Diabetes Care 1996; 19:920-6.

51. Buchanan TA, Xiang AH, Peters RK, Kjos SL, Marroquin A Goico J, et al. Preservation of pancreatic B-cell function and prevention of type 2 diabetes by pharmacological treatment of insulin resistance in high-risk Hispanic women. Diabetes 2002;51:2796-803.

52. Knowler WC, Hamman RF, Edelstein SL, Barrett-Connor E, Ehrmann DA, Walker EA, et al; Diabetes Prevention Program Research Group. Prevention of type 2 diabetes with troglitazone in the Diabetes Prevention Program. Diabetes 2005:54:1150-6.

53. Durbin RJ. Thiazolidinedione therapy in the prevention/ delay of type 2 diabetes in patients with impaired glucose tolerance and insulin resistance. Diabetes Obes Metab 2004;6:280-5.

54. Miyazaki Y, Mahankali A, Matsuda M, Glass L, Mahankali S, Ferrannini $E$, et al. Improved glycemic control and enhanced insulin sensitivity in type 2 diabetic subjects treated with pioglitazone. Diabetes Care 2001:24:710-9.

55. Torgerson JS, Hauptman J, Boldrin MN, Sjostrom L. Xenical in the prevention of diabetes in obese subjects (XENDOS) study: a randomized study of orlistat as an adjunct to lifestyle changes for the prevention of type 2 diabetes in obese patients. Diabetes Care 2004; 27:155-61.

56. Heymsfield SB, Segal KR, Hauptman J, Lucas CP, Boldrin $M N$, Rissanen $A$, et al. Effects of weight loss with orlistat on glucose tolerance and progression to type 2 diabetes in obese adults. Arch Intern Med 2000; 160:1321-6.

57. Padwal R, Laupacis A. Antihypertensive therapy and incidence of type 2 diabetes: a systematic review. Diabetes Care 2004;27:247-55.
58. Savage PH, Pressel SL, Curb D, Schron EB, Applegate WB, Black HR, et al. Influence of long-term, low-dose, diuretic-based antihypertensive therapy on glucose, lipid, uric acid, and potassium levels in older men and women with isolated systolic hypertension. Arch Intern Med 1998; 158:741-75

59. Fletcher A, Amery A, Birkenhager W, Bulpitt C, Clement $D$, de Leeuw $P$, et al. Risks and benefits in the trial of the European Working Party on High Blood Pressure in the Elderly. J Hypertens 1991;9:225-30.

60. Yusuf S, Gerstein H, Hoogwerf B, Pogue J, Bosch J, Wolffenbuttel BHR, et al. Ramipril and the development of diabetes. JAMA 2001;286:1882-5.

61. Hansson L, Lindholm LH, Niskanen L, Lanke J, Hedner T, Niklason $A$, et al. Effect of angiotensin-convertingenzyme inhibition compared with conventional therapy: the Captopril Prevention Project (CAPP) randomized trial. Lancet 1999;35:611-6.

62. Pfeffer MA, Swedberg K, Granger CB, Held P, McMurray JJV, Michelson EL, et al. Effects of candesartan on mortality and morbidity in patients with chronic heart failure: the CHARM-Overall programme. Lancet 2003;362:759-66.

63. Lithell H, Hansson L, Skoog I, Elmfeldt D, Hofman A, Olofsson B, et al. The Study on Cognition and Prognosis in the Elderly (SCOPE): principle results of a randomized double-blind intervention trial. J Hypertens 2003: $21: 875-86$.

64. Lindholm LH, Ibsen $\mathrm{H}$, Borch-Johnsen $\mathrm{K}$, Olsen $\mathrm{MH}$, Wachtell K, Dahlof B, et al. Risk of new-onset diabetes in the Losartan Intervention For Endpoint reduction in hypertension study. J Hypertension 2002;20:1879-86.

65. Pepine CJ, Handberg EM, Cooper-DeHoff RM, Marks $R G$, Kowey $P$, Messerli FH, et al. A calcium antagonist vs. a non-calcium antagonist hypertension treatment strategy for patients with coronary artery disease: the International Verapamil-Trandolapril Study (INVEST): a randomized controlled trial. JAMA 2003;290:2805-16.

66. Kanaya AM, Herrington D, Vittinghoff E, Lin F, Grady D, Bittner $\mathrm{V}$, et al; Heart and Estrogen/progestin Replacement Study. Glycemic effects of postmenopausal hormone therapy: the Heart and Estrogen/progestin Replacement Study. A randomized, double-blind, placebo-controlled trial. Ann Intern Med 2003; 138:1-9.

67. Julius $S$, Kjeldsen SE, Weber M, Brunner HR, Ekman S, Hansson L, et al; the VALUE Trial Group. Outcomes in hypertensive patients at high cardiovascular risk treated with regimens based on valsartan or amlodipine: the VALUE randomized trial. Lancet 2004;363:2022-31.

68. Freeman DJ, Norrie J, Sattar N, Neely DG, Cobbe SM, Ford I, et al. Pravastatin and the development of diabetes mellitus: evidence for a protective treatment effect in the West of Scotland Coronary Prevention Study. Circulation 2001; 103:357-62.

69. Keech A, Colquhoun D, Best J, Kirby A, Simes RJ, Hunt D, et al. Secondary prevention of cardiovascular events with long-term pravastatin in patients with diabetes or impaired fasting glucose. Diabetes Care 2003; 26:2713-21 
70. Heart Protection Study Collaborative Group. MRC/BHF Heart Protection Study of cholesterol-lowering with simvastatin in 5963 people with diabetes: a randomized placebo-controlled trial. Lancet 2003;361:2005-16.

71. Sever PS, Dahlof B, Poulter NR, Wedel H, Beevers G, Caulfield $M$, et al. Prevention of coronary and stroke events with atorvastatin in hypertensive patients who have average or lower-than-average cholesterol concentrations, in the Anglo-Scandinavian Cardiac Outcomes Trial - Lipid Lowering Arm (ASCOT-LLA): a multicentre randomised controlled trial. Lancet 2003;361:1149-58.

72. Tenenbaum A, Motro M, Fisman EZ, Schwammenthal $E$ Adler Y, Goldenberg I, et al. Peroxisome proliferatorsactivated receptor ligand Bezafibrate for prevention of type 2 diabetes mellitus in patients with coronary artery disease. Circulation 2004; 109:2197-202.

73. Manson JE, Rimm EB, Colditz GA, Willett WC, Nathan DM. Arky RA, et al. A prospective study of postmenopausal estrogen therapy and subsequent incidence of non-insulin-dependent diabetes mellitus. Ann Epidemiol 1992:2:665-73
74. Sjostrom CD, Lissner L, Wedel H, Sjostrom L. Reduction in incidence of diabetes, hypertension and lipid disturbances after intentional weight loss induced by bariatric surgery: the SOS intervention study. Obesity Res 1999; 7:477-84.

75. Sjöström CD, Peltonen $M$, Wedel $H$, Sjöström L. Differentiated long-term effects of intentional weight loss on diabetes and hypertension. Hypertension 2000: 36:20-5.

76. Pories WJ, MacDonald KG, Morgan EJ, Sinha MK, Dohm GL, Swanson MS, et al. Surgical treatment of obesity and its effect on diabetes: 10-y follow-up. Am J Clin Nutr 1992;55:582S-5.

\section{Endereço para correspondência:}

Ruy Lyra

Rua Visconde de Albuquerque 137

50610-090 Recife, PE

Fax: (81) 3445-1145

E-mail: ruylyra@hotlink.com.br 